\title{
Isomer selective infrared spectroscopy of neutral metal clusters
}

\author{
André Fielicke \\ Fritz-Haber-Institut der Max-Planck-Gesellschaft, Faradayweg 4-6, D-14195 Berlin, Germany \\ Christian Ratsch \\ Fritz-Haber-Institut der Max-Planck-Gesellschaft, Faradayweg 4-6, D-14195 Berlin, Germany and \\ Department of Mathematics, University of California at Los Angeles, Los Angeles, California 90095-1555
}

Gert von Helden and Gerard Meijer

Fritz-Haber-Institut der Max-Planck-Gesellschaft, Faradayweg 4-6, D-14195 Berlin, Germany

(Received 18 January 2005; accepted 26 January 2005; published online 4 March 2005)

\begin{abstract}
We report experimental infrared spectra of neutral metal clusters in the gas phase. Multiple photon dissociation of the argon complexes of niobium clusters is used to obtain vibrational spectra in the $80-400 \mathrm{~cm}^{-1}$ region. The observed spectra for $\mathrm{Nb}_{9} \mathrm{Ar}_{n}(n=1-4)$ are different for different values of $n$. This is explained by the presence of two isomers of $\mathrm{Nb}_{9}$ that have different affinities towards $\mathrm{Ar}$ and the isomer specific infrared spectra are obtained. The structures of the isomers are determined by comparing the observed spectra with the outcome of density-functional theory calculations. (C) 2005 American Institute of Physics. [DOI: 10.1063/1.1872834]
\end{abstract}

The knowledge on the geometric and electronic structure of a cluster forms the basis for the understanding of its physical and chemical properties. Geometric structure determination of metal clusters in the gas phase is difficult and for systems where isomers are present, the structure determination is even more challenging. For metal clusters, the coexistence of different isomeric structures has been experimentally revealed using mainly two methods: (i) investigation of the kinetics with reactant molecules, where bi- or multipleexponential reaction kinetics are signatures for the presence of isomers with different reactivities, ${ }^{1-5}$ or (ii) (ion) mobility measurements, where isomers with different collision cross sections can be separated and detected via their different arrival time distributions. ${ }^{6-8}$ Further evidence for metal cluster isomers is obtained from the measurements of magnetic moments, ${ }^{9}$ from unimolecular photodissociation dynamics, ${ }^{10}$ and from photoelectron spectroscopy. ${ }^{11}$ Very recently, optical spectroscopy on matrix deposited silver clusters allowed for the identification of the presence of silver cluster isomers. ${ }^{12}$

In principle, vibrational spectroscopy can be used to obtain structural information on metal clusters, but until recently, this approach was limited to selected small clusters that have mainly been investigated via matrix isolation spectroscopy ${ }^{13}$ or to clusters where vibrational structure in electronic excitation spectra has been found. ${ }^{14-18}$ For cationic metal clusters, we have recently demonstrated that farinfrared (vibrational) resonance enhanced multiple photon dissociation (FIR-MPD) spectroscopy of their complexes with rare gas atoms is a suitable method to obtain their vibrational spectra, and to thus get direct information on their geometric structures. ${ }^{19}$ Here we report on FIR-MPD spectroscopic studies of neutral metal clusters. We are focusing in particular on $\mathrm{Nb}_{9}$, to demonstrate that isomer specific vibrational spectra can be obtained. The structures of the isomers are determined by comparison of the observed FIR-MPD spectra to calculated IR absorption spectra as obtained from density-functional theory calculations.

The experimental set-up has been described elsewhere ${ }^{19}$ and only a brief description is given here. Argon complexes of niobium clusters are produced in a pulsed laser ablation cluster source that is partly cooled to $\approx 80 \mathrm{~K}$. As carrier gas, a mixture of $20 \%-40 \%$ Argon in Helium is used. The binding of the rare gas atoms to neutral metal clusters is much weaker than the binding to cationic metal clusters and complex formation is, therefore, by far more sensitive to source parameters such as timings, partial pressures, and fluence of the vaporization laser. The cluster complexes are detected in a time-of-flight mass spectrometer after ionization with either an ArF excimer laser (6.42 eV/photon) or a frequency-doubled dye laser. As the ionization potentials (IPs) for niobium clusters are typically in the 4.6-5.5 eV range $^{20,21}$ and as the IPs of their Ar complexes will be slightly lower, the $6.42 \mathrm{eV}$ photon energy allows for one photon ionization of all clusters and their Ar complexes. The fluence of the ArF laser is kept sufficiently low to prevent absorption of multiple UV photons that would lead to fragmentation of the weakly bound complexes. With this method, niobium cluster complexes containing up to four Ar atoms have been detected. Alternatively, the frequency doubled output of a dye laser is used for the more selective ionization near the threshold (see below). The molecular beam is overlapped with a counter-propagating IR laser beam emitted by the Free Electron Laser for Infrared Experiments (FELIX). ${ }^{22}$ When the IR light is resonant with a transition in the cluster complex, the complex can absorb several photons and subsequently evaporate off one or more rare gas atoms. The resulting abundance changes of the rare gas complexes are recorded as a function of the IR frequency. Details on the normalization of the spectra to the laser intensity and on the conversion of the depletion spectra into absorption spectra are described elsewhere. ${ }^{19}$ 

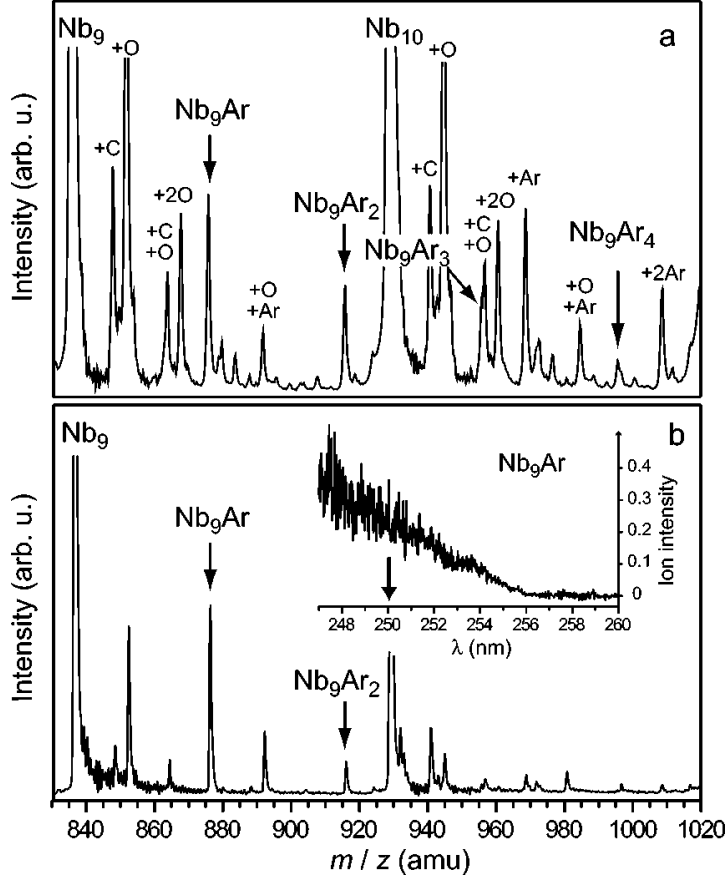

FIG. 1. Mass spectra in the mass range of $\mathrm{Nb}_{9} \mathrm{Ar}_{n}(n=0-4)$ recorded using (a) $6.42 \mathrm{eV}$ photons and (b) $4.96 \mathrm{eV}$ photons for the ionization. The conditions under which spectrum (b) is recorded are optimized for $\mathrm{Nb}_{9} \mathrm{Ar}$. At 4.96 $\mathrm{eV}(250 \mathrm{~nm})$ the Ar complexes of only one isomer of $\mathrm{Nb}_{9}$ can be ionized. A measurement of the ionization threshold is shown in the inset.

Figure 1 shows parts of the typical mass spectra in the range of $\mathrm{Nb}_{9} \mathrm{Ar}_{n}(n=0-4)$. In the spectrum in Fig. 1(a) the intensities of the rare gas complexes are usually a factor of $\approx 50$ lower than those of the bare metal clusters. Additional peaks can be assigned to oxide and carbide contaminations. For $n=3$ and 4 the peaks of the complexes overlap with those of the contaminants. However, this does not lead to interferences in the IR spectra of $\mathrm{Nb}_{9} \mathrm{Ar}_{n}$ since only the rare gas complexes can be dissociated.

Far-infrared absorption spectra of free neutral niobium clusters are measured via multiple photon dissociation of the $\mathrm{Nb}_{m} \mathrm{Ar}_{n}$ complexes in the $80-400 \mathrm{~cm}^{-1}$ region. Using $\mathrm{ArF}$ ionization for detection, spectra are recorded for clusters containing five to more than $20 \mathrm{Nb}$ atoms and different numbers of Ar atoms. These results will be reported elsewhere, but important for the discussion here is that for almost all of the clusters the observed FIR-MPD spectra are invariant with the number of attached Ar atoms. Only small shifts of the absorption peaks (on the order of $1-3 \mathrm{~cm}^{-1}$ ) and slight broadenings are observed for a given $\mathrm{Nb}_{m}$ cluster with an increasing number of attached $\mathrm{Ar}$ atoms.

A special case are the $\mathrm{Nb}_{9} \mathrm{Ar}_{n}(n=1-4)$ complexes. Their FIR-MPD spectra are shown in Fig. 2. For $\mathrm{Nb}_{9} \mathrm{Ar}_{n}$ $(n=1-4)$ absorption features are only found in the $180-300 \mathrm{~cm}^{-1}$ region. In the FIR-MPD spectrum of $\mathrm{Nb}_{9} \mathrm{Ar}$ four main bands are found at $\approx 195,202,252$ (P3), and at $280 \mathrm{~cm}^{-1}$ (P4). In addition, a minor peak can be identified around $215 \mathrm{~cm}^{-1}$ (P2) and there is a shoulder on the low frequency side of the peak P3. With increasing number of Ar atoms in the complex, the double peak around $200 \mathrm{~cm}^{-1}$

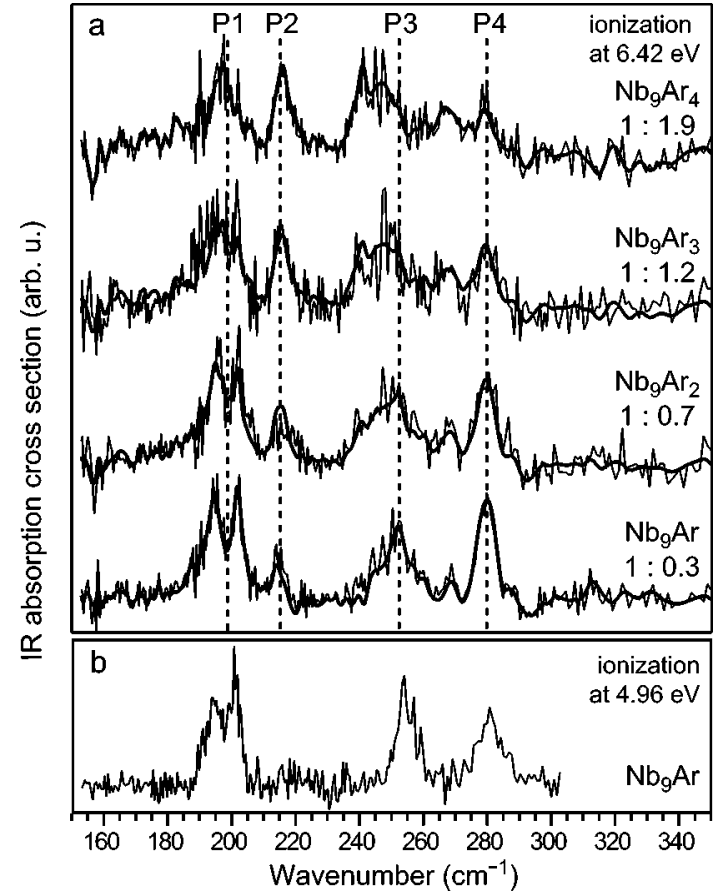

FIG. 2. (a) Far-IR multiple photon dissociation (FIR-MPD) spectra of $\mathrm{Nb}_{9} \mathrm{Ar}_{n}(n=1-4)$. The neutral clusters are ionized with $6.42 \mathrm{eV}$ photons. The major features in the spectra are indicated with P1-P4. Reconstructed spectra of mixtures of the two isomers in the given ratios (intensities of isomer 1 to isomer 2) are shown overlayed with the experimental spectra. (b) FIR-MPD spectrum of $\mathrm{Nb}_{9} \mathrm{Ar}$ as obtained by ionization using $250 \mathrm{~nm}$ radiation.

merges into a single peak (P1), the peak $\mathrm{P} 2$ as well as the shoulder on $\mathrm{P} 3$ gain in intensity whereas the originally dominating peaks decrease in intensity.

The strong dependence of the FIR-MPD spectra of $\mathrm{Nb}_{9} \mathrm{Ar}_{n}$ on the number of Ar atoms can be explained by the presence of (at least) two isomers of $\mathrm{Nb}_{9}$ that have different affinities towards the Ar atoms. To be more precise, the relative affinity of these $\mathrm{Nb}_{9}$ isomers towards Ar must gradually change with the number $n$ of Ar atoms. This then leads to a change in the relative contribution of the $\mathrm{Nb}_{9}$ isomers to the spectra of the $\mathrm{Nb}_{9} \mathrm{Ar}_{n}$ complexes as a function of $n$, and can explain the observations. The presence of (at least) two isomers of $\mathrm{Nb}_{9}$ has been concluded earlier from the observed biexponential reaction kinetics with hydrogen or nitrogen ${ }^{1-3}$ and the different IPs for the isomers have been experimentally determined to be 4.92 and $5.20 \mathrm{eV}^{20}{ }^{20}$ Optical absorption spectra for $\mathrm{Nb}_{9}$ have been recorded using photodissociation of their rare gas cluster complexes ${ }^{23,24}$ and the photoionization threshold behavior of these complexes has been investigated as well. ${ }^{25}$ Also in these studies, differences in the propensities to bind Ar atoms have been found for the isomers of $\mathrm{Nb}_{9}$.

Alternatively, one might explain the observed changes in the FIR-MPD spectra of $\mathrm{Nb}_{9} \mathrm{Ar}_{n}$ as a function of $n$ by assuming that there is only one $\mathrm{Nb}_{9}$ isomer but that the oscillator strengths of the different vibrational modes are differently affected by complexing the metal cluster with an increasing number of Ar atoms. This explanation seems highly unlikely, however, as we have not observed any significant influence of the rare gas atoms on the FIR-MPD spectra for other clusters. 
The difference in the IPs of the two $\mathrm{Nb}_{9}$ isomers enables the selective ionization of the isomer with the lowest IP using near threshold ionization. The onset of ionization for $\mathrm{Nb}_{9} \mathrm{Ar}$ is shown in the inset of Fig. 1(b). An ionization threshold of $4.845 \pm 0.002 \mathrm{eV}$ (under field-free conditions) is found, in agreement with previous findings. ${ }^{25}$ Figure 1(b) shows a mass spectrum that has been obtained using near threshold ionization with $250 \mathrm{~nm}$ radiation. Under the assumption that the Ar induced shift of the IPs is approximately the same for both isomers $(\approx 0.07 \mathrm{eV})$, then the $\mathrm{Ar}$ complex of the second isomer should have an IP of $\approx 5.13 \mathrm{eV}$, well above the photon energy at $250 \mathrm{~nm}$.

The FIR-MPD spectrum of $\mathrm{Nb}_{9} \mathrm{Ar}$ measured with near threshold ionization is shown in Fig. 2(b). Only the Ar complex of one $\mathrm{Nb}_{9}$ isomer can be ionized and therefore this spectrum can be assigned to the isomer with the lowest IP (isomer 1). This FIR-MPD spectrum is quite similar to the FIR-MPD spectrum of $\mathrm{Nb}_{9} \mathrm{Ar}$ measured with $\mathrm{ArF}$ ionization. Therefore, we conclude that isomer $\mathbf{1}$ has a higher propensity for binding a single Ar atom than isomer 2. The observed changes in the FIR-MPD spectra indicate that with increasing number of Ar atoms in the complex the abundance of isomer $\mathbf{1}$ decreases relative to the abundance of isomer $\mathbf{2}$.

The spectra of the individual isomers can be extracted from the spectra of the isomeric mixture by iterative subtractive deconvolution. As a starting point, it is assumed that the spectrum of $\mathrm{Nb}_{9} \mathrm{Ar}$ is dominated by isomer $\mathbf{1}$, whereas isomer 2 dominates the spectrum of $\mathrm{Nb}_{9} \mathrm{Ar}_{4}$. The deconvolution has been applied after smoothing the data with a three point fast Fourier transform filter. No information from the FIRMPD spectrum as obtained after selective ionization has been used in this approach. The thus obtained isomer selective FIR-MPD spectra are shown in Fig. 3(a). Combinations of these spectra in the appropriate intensity ratio are shown overlayed with the observed spectra in Fig. 2(a). The intensity ratio changes from 1:0.3 to 1:1.9 when $n$ increases from one to four, but these numbers only indicate the relative abundance ratio of the two isomers as a function of $n$ since the absolute IR absorption intensities and the ionization efficiencies are not known. The FIR-MPD spectrum of isomer 1 as extracted from the spectra of the isomeric mixture is very similar to the spectrum that is obtained using selective ionization, shown once more in Fig. 3(b).

Density-functional theory (DFT) calculations have been performed to find the energetically most preferred structures for $\mathrm{Nb}_{9}$, and their vibrational spectra have been calculated. We have used the $\mathrm{DMOL}^{3}$ code $^{26}$ and the generalized gradient approximation $(\mathrm{GGA})^{27}$ for the exchange correlation functional. More details of the computational procedure will be given elsewhere. ${ }^{28}$ As a check, for $\mathrm{Nb}_{2}$ this approach yields a calculated vibrational frequency of $445 \mathrm{~cm}^{-1}$, close to the experimental value of $425 \mathrm{~cm}^{-1} .{ }^{31}$ We tested a large number of different geometric structures for $\mathrm{Nb}_{9}$, including some that have been suggested previously in the literature. ${ }^{29,30}$ Moreover, we tested a number of geometric distortions of these structures, and calculated the energy and the IR absorption spectra for different spin states. The vibrational frequencies presented here are not scaled.

In Fig. 3(c) the results of the calculations for three dif-

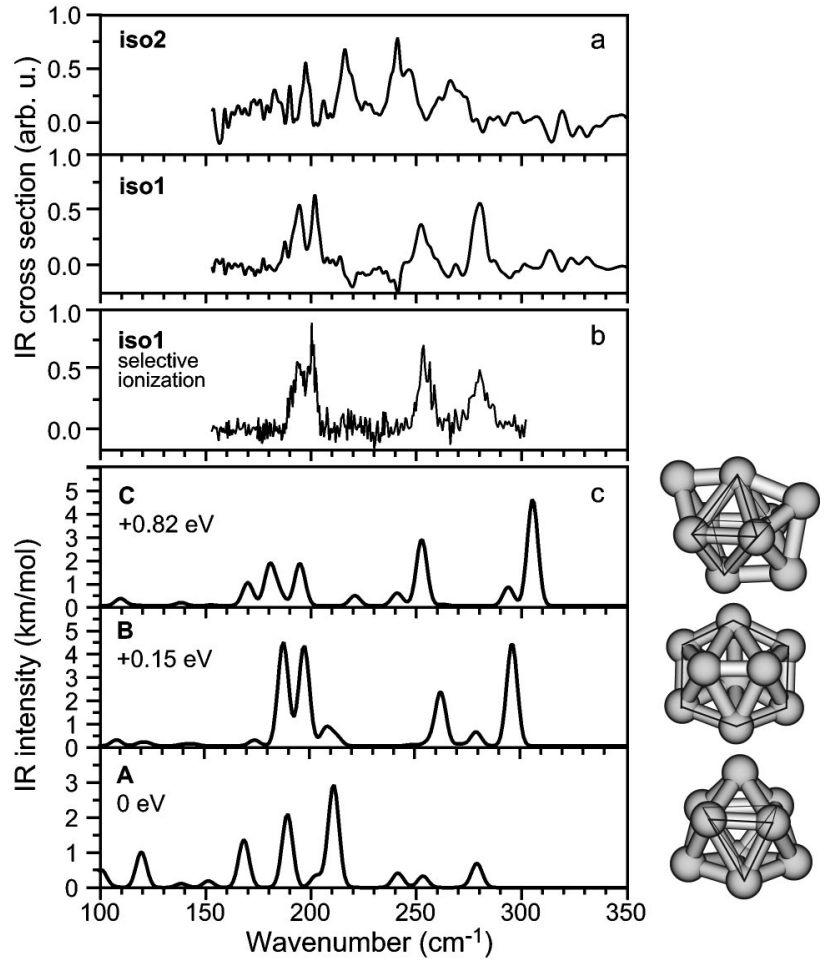

FIG. 3. Isomer selective FIR-MPD spectra. Panel (a) shows the isomer selective FIR-MPD spectra as extracted out of the spectra of $\mathrm{Nb}_{9} \mathrm{Ar}_{n}$ (shown in Fig. 2) for each of the two $\mathrm{Nb}_{9}$ isomers 1 and 2. Panel (b) shows the FIR-MPD spectrum of isomer $\mathbf{1}$ as obtained by selective ionization of this isomer with $250 \mathrm{~nm}$ radiation. Panel (c) shows the calculated IR absorption spectra for three low energy isomers of $\mathrm{Nb}_{9}$ labeled $\mathbf{A}, \mathbf{B}$, and $\mathbf{C}$. The calculated spectra are folded with a Gaussian line shape function with a width (FWHM) of $5 \mathrm{~cm}^{-1}$.

ferent structures of $\mathrm{Nb}_{9}$ are shown. The lowest energy structure $\mathbf{A}$ can be described as a distorted triply capped trigonal prism. ${ }^{32}$ Structure $\mathbf{B}$ is only $0.15 \mathrm{eV}$ higher in energy and is a nonplanar hexagon with a dimer above and an atom below the hexagon. The next stable structure $\mathbf{C}$, a distorted triply capped octahedron, is noticeably higher in energy $(0.82 \mathrm{eV})$. All these structures correspond to a doublet electronic state and structures with higher multiplicity are found to be significantly higher in energy. The IR spectrum of structure B nicely agrees with the experimental spectrum of isomer $\mathbf{1}$; the band positions as well as the IR intensities are reproduced rather well. There is considerably less agreement between the observed spectrum of isomer $\mathbf{2}$ and the calculated IR spectra of either structure A or structure C. Nevertheless, the comparison of the spectra suggests that isomer $\mathbf{2}$ may have structure $\mathbf{A}$; it is the energetically lowest structure, and there is some agreement between the spectra. The two calculated bands at $189 \mathrm{~cm}^{-1}$ and at $211 \mathrm{~cm}^{-1}$ can be related to features in the experimental spectrum. Below $200 \mathrm{~cm}^{-1}$ the experimental spectrum is noisy and not well-resolved, preventing a firm conclusion on the presence or absence of the band calculated at $168 \mathrm{~cm}^{-1}$ in the experimental spectrum. The calculations further predict IR bands at 241, 253, 278, and $279 \mathrm{~cm}^{-1}$. This is in agreement with the band positions in the experimental spectrum, but the intensities do not match. One has to keep in mind, however, that the experimental spectra are obtained by multiple photon absorption 
followed by dissociation, and that intensities in FIR-MPD spectra can differ considerably from those in linear FIR absorption spectra. Further evidence for the assignments proposed here is derived from the calculation of the (adiabatic) ionization energies. We find values of $5.13 \mathrm{eV}$ for structure A and $4.98 \mathrm{eV}$ for structure $\mathbf{B}$ that are in good agreement with the experimental values. The assignment of isomer $\mathbf{1}$, the isomer with the lowest IP, to structure $\mathbf{B}$ seems quite certain. The assignment for isomer $\mathbf{2}$ is less certain, and we cannot exclude that this isomer has yet another structure, that has not been found in the calculations thus far.

Finally, the question remains to be answered why these two $\mathrm{Nb}_{9}$ isomers show different Ar binding properties. The calculations show that structure $\mathbf{B}$ has a dipole moment of $0.70 \mathrm{D}$, considerably higher than the $0.12 \mathrm{D}$ dipole moment of structure A. This can explain the observed preferred binding of the first (two) Ar atom(s) to isomer 1. However, the large dipole moment of structure $\mathbf{B}$ is mainly caused by the presence of the single $\mathrm{Nb}$ atom below the hexagon unit that carries a significant positive partial charge. It is likely, therefore, that after the first (two) Ar atoms(s) are attached to this side of the $\mathrm{Nb}_{9}$ isomer, the next $\mathrm{Ar}$ atoms either have to attach to a less favorable side of the complex or they attach to the same side of the complex where they then experience a strongly shielded dipole. In either case, this can well explain the observed (relative) decrease in affinity for Ar atoms with increasing number of Ar atoms for isomer 1.

In conclusion, we have shown that vibrational spectra of neutral metal clusters can be measured via multiple photon dissociation spectroscopy of their rare gas complexes using tunable radiation in the far-infrared. In special cases even isomer specific spectra can be obtained. Together with theoretical studies, these spectra enable the structure of metal cluster isomers to be unraveled.

We acknowledge stimulating discussions with D. M. Rayner and M. Scheffler. This work is part of the research program of the "Stichting voor Fundamenteel Onderzoek der Materie" (FOM), which is financially supported by the "Nederlandse Organisatie voor Wetenschappelijk Onderzoek" (NWO). Financial support from the EU IHP Research Training Network (Delayed Ionization and Competing Cooling Mechanisms in Atomic Clusters) is gratefully acknowledged. We also thank Accelrys for providing the $\mathrm{DMOL}^{3}$ software to the California NanoSystems Insitute.
${ }^{1}$ M. R. Zakin, R. O. Brickman, D. M. Cox, and A. Kaldor, J. Chem. Phys. 88, 3555 (1988)

${ }^{2}$ Y. Hamrick et al., J. Chem. Phys. 88, 4095 (1988); J. Phys. Chem. 93, 6494 (1989).

${ }^{3}$ A. Bérces et al., J. Chem. Phys. 108, 5476 (1998).

${ }^{4}$ J. Ho, L. Zhu, E. K. Parks, and S. J. Riley, J. Chem. Phys. 99, 140 (1993).

${ }^{5}$ C. Berg et al., Chem. Phys. 262, 143 (2000).

${ }^{6}$ G. von Helden, M.-T. Hsu, P. R. Kemper, and M. T. Bowers, J. Chem. Phys. 95, 3835 (1991)

${ }^{7}$ M. F. Jarrold and J. E. Bower, J. Chem. Phys. 98, 2399 (1993); A. A. Shvartsburg and M. F. Jarrold, Phys. Rev. A 60, 1235 (1999).

${ }^{8}$ P. Weis, T. Bierweiler, E. Vollmer, and M. M. Kappes, J. Chem. Phys. 117, 9293 (2002).

${ }^{9}$ J. P. Bucher, D. C. Douglass, and L. A. Bloomfield, Phys. Rev. Lett. 66, 3052 (1991)

${ }^{10}$ M. Vogel, K. Hansen, and L. Schweikhard, Int. J. Mass. Spectrom. 233, 117 (2004)

${ }^{11}$ H. Kietzmann et al., Phys. Rev. Lett. 77, 4528 (1996); J. Chem. Phys. 109, 2275 (1998).

${ }^{12}$ C. Sieber et al., Phys. Rev. A 70, 041201 (2004).

${ }^{13}$ J. R. Lombardi and B. Davis, Chem. Rev. (Washington, D.C.) 102, 2431 (2002).

${ }^{14}$ M. F. Jarrold and K. M. Creegan, Chem. Phys. Lett. 166, 116 (1990).

${ }^{15}$ K. J. Taylor et al., J. Chem. Phys. 93, 7515 (1990).

${ }^{16}$ G. F. Ganteför, D. M. Cox, and A. Kaldor, J. Chem. Phys. 96, 4102 (1992); H. Handschuh, G. Ganteför, and W. Eberhardt, Rev. Sci. Instrum. 66, 3838 (1995).

${ }^{17}$ D. S. Yang, A. M. James, D. M. Rayner, and P. A. Hackett, Chem. Phys. Lett. 231, 177 (1994).

${ }^{18}$ T. P. Marcy and D. G. Leopold, Int. J. Mass. Spectrom. 196, 653 (2000).

${ }^{19}$ A. Fielicke et al., Phys. Rev. Lett. 93, 023401 (2004).

${ }^{20}$ M. B. Knickelbein and S. Yang, J. Chem. Phys. 93, 1476 (1990); 93, 5760 (1990).

${ }^{21}$ K. Athanassenas et al., Chem. Phys. Lett. 213, 105 (1993).

${ }^{22}$ D. Oepts, A. F. G. van der Meer, and P. W. van Amersfoort, Infrared Phys. Technol. 36, 297 (1995).

${ }^{23}$ M. B. Knickelbein and W. J. C. Menezes, Phys. Rev. Lett. 69, 1046 (1992); W. J. C. Menezes and M. B. Knickelbein, J. Chem. Phys. 98, 1856 (1993).

${ }^{24}$ B. A. Collings, K. Athanassenas, D. M. Rayner, and P. A. Hackett, Z. Phys. D: At., Mol. Clusters 26, 36 (1993).

${ }^{25}$ M. B. Knickelbein and W. J. C. Menezes, Chem. Phys. Lett. 184, 433 (1991); J. Phys. Chem. 96, 6611 (1992).

${ }^{26}$ B. Delley, J. Chem. Phys. 92, 508 (1990).

${ }^{27}$ J. P. Perdew, K. Burke, and M. Ernzerhof, Phys. Rev. Lett. 77, 3865 (1996).

${ }^{28}$ C. Ratsch, A. Fielicke, A. Kirilyuk, J. Behler, G. von Helden, G. Meijer, and M. Scheffler, J. Chem. Phys. (in press).

${ }^{29}$ H. Grönbeck, A. Rosén, and W. Andreoni, Phys. Rev. A 58, 4630 (1998).

${ }^{30}$ V. Kumar and Y. Kawazoe, Phys. Rev. B 65, 125403 (2002).

${ }^{31}$ A. M. James, P. Kowalczyk, R. Fournier, and B. Simard, J. Chem. Phys. 99, 8504 (1993).

${ }^{32}$ We have marked in Fig. 3(c) the core parts of the structures for better recognizability of the structural elements. Structural descriptions in terms of other elements are equally possible. 